\title{
In Vivo Vascularization of Anisotropic Channeled Porous Polylactide- Based Capsules for Islet Transplantation: The Effects of Scaffold Architecture and Implantation Site
}

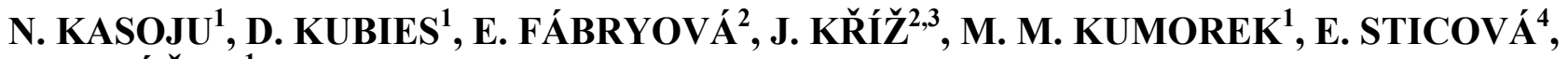 \\ F. RYPÁČEK ${ }^{1}$
}

${ }^{1}$ Department of Biomaterials and Bioanalogous Polymer Systems, Institute of Macromolecular Chemistry, Academy of Sciences of the Czech Republic, Prague, Czech Republic, ${ }^{2}$ Center of Experimental Medicine, and ${ }^{3}$ Department of Diabetes, Institute for Clinical and Experimental Medicine, Prague, Czech Republic, ${ }^{4}$ Department of Pathology, Third Faculty of Medicine, Charles University and Faculty Hospital of Kralovske Vinohrady, Prague, Czech Republic

Received June 9, 2015

Accepted July 16, 2015

\section{Summary}

The replacement of pancreatic islets for the possible treatment of type 1 diabetes is limited by the extremely high oxygen demand of the islets. To this end, here we hypothesize to create a novel extra-hepatic highly-vascularized bioartificial cavity using a porous scaffold as a template and using the host body as a living bioreactor for subsequent islet transplantation. Polylactide-based capsular-shaped anisotropic channeled porous scaffolds were prepared by following the unidirectional thermallyinduced phase separation technique, and were implanted under the skin and in the greater omentum of Brown Norway rats. Polyamide mesh-based isotropic regular porous capsules were used as the controls. After 4weeks, the implants were excised and analyzed by histology. The hematoxylin and eosin, as well as Masson's trichrome staining, revealed a) low or no infiltration of giant inflammatory cells in the implant, b) minor but insignificant fibrosis around the implant, c) guided infiltration of host cells in the test capsule in contrast to random cell infiltration in the control capsule, and d) relatively superior cell infiltration in the capsules implanted in the greater omentum than in the capsules implanted under the skin. Furthermore, the anti-CD31 immunohistochemistry staining revealed numerous vessels at the implant site, but mostly on the external surface of the capsules. Taken together, the current study, the first of its kind, is a significant step-forward towards engineering a bioartificial microenvironment for the transplantation of islets.

\begin{abstract}
Key words
Diabetes - Islet transplantation - Subcutaneous - Omentum • Vascularization - Biomaterials - Thermally induced phase separation • Polylactide • Anisotropy • Channeled pores • Guided cell infiltration $\bullet$ Histology

\section{Corresponding authors}

N. Kasoju and D. Kubies, Department of Biomaterials and Bioanalogous Polymer Systems, Institute of Macromolecular Chemistry, Academy of Sciences of the Czech Republic, Prague 162 06, Czech Republic. Fax: +420 296809 410. Email: kasoju@imc.cas.cz and kubies@imc.cas.cz; J. Křriž, Department of Diabetes, Institute for Clinical and Experimental Medicine, Prague 140 21, Czech Republic. Fax: +420 236053 127. E-mail: jkri@ikem.cz
\end{abstract}

\section{Introduction}

The autoimmune destruction of beta cells in the pancreatic islets of Langerhans leads to total insulin deficiency and type 1 diabetes mellitus (T1DM). The ensuing lack of insulin results in increased glucose levels, which is associated with chronic complications of high morbidity and mortality among young adults who are in a productive age. Currently, the sole treatment for T1DM is exogenous insulin injections or insulin pumps in combination with glucose monitoring meters; But the complexity of glucose control often results in hypo- or 
hyper- glycaemia and associated complications. Whole pancreas transplantation has been explored as an alternative strategy, but the lack of adequate donor organs and the scale of surgery are reportedly the major limiting factors (Vardanyan et al. 2010). In a breakthrough study, investigators from the University of Alberta in the Canadian city of Edmonton reported intra-hepatic portal islet transplantation in combination with a steroid-free immuno-suppression cocktail (Shapiro et al. 2000). This approach, known as the Edmonton protocol, has gained popularity owing to its minimally-invasive operation and reduced post-operative complications as compared to whole pancreases transplantation. Despite some successful results, the Edmonton protocol is still not yet used in routine clinical practice as it suffers from procedure-related issues such as portal vein bleeding, instant blood-mediated inflammatory reactions including portal vein thrombosis, and post-transplant later complications such as loss of islets due to glucotoxicity, lipotoxicity, toxicity by other substances just absorbed from the gastrointestinal tract and permanent exposure to hypoxic conditions (Korsgren et al. 2008, Leitão et al. 2008). Both the procedure-related and the post-transplant impediments were associated with nothing but the site itself.

To overcome these intra-hepatic portal vein associated issues, a variety of alternative extra-hepatic sites were investigated, including the pancreas (Stagner et al. 2007), kidney capsule (Carlsson et al. 2000), spleen (Wohlrab et al. 1988), gastric submucosa (Caiazzo et al. 2007), bone marrow (Cantarelli et al. 2009), striated muscle (Svensson et al. 2011) and the anterior chamber of the eye (Perez et al. 2011). However, modifying an existing tissue or organ involves extensive invasive procedures, interferes with normal functioning and therefore carries a high risk of unwanted pathological conditions. Recently, attempts have been undertaken to explore several biomaterials for potential application in islet transplantation (Bratlie et al. 2012). But, the majority of these investigations have been dominated by islet encapsulation approaches, such as nanoencapsulation using thin conformal coatings (Krol et al. 2006), micro-encapsulation within hydrogels (Ma et al. 2013) and macro-encapsulation inside hollow fibers or other macro-devices (Tatarkiewicz et al. 1999). All the nano- and micro-encapsulation approaches were solely aimed at protecting the islets from allo- and auto-immune attacks, without offering any adequate blood perfusion of the extra-hepatic transplantation site. On the other hand, the macro-encapsulation strategies demonstrated the creation of an artificial housing for the islets, but again with a focus on immuno-protection. Moreover, the total mass of the transplanted tissue was higher than the intraportal transplantation; and in addition, the long-term viability and clinical success of these approaches has not yet been reported. One of the bottlenecks in such immuno-protective macro-encapsulation strategies is the large diffusion distance from neighboring blood vessels to the encapsulated islets and consequent ischemiainduced loss of viable cells.

As outlined above, when bioengineering an artificial site for the transplantation of pancreatic islets, a key challenge, more vital than immuno-protection, is to ensure the continuous supply of oxygen and nutrients and thereby to allow long-term islet survival and functioning. To this end, for the first time, here we hypothesize to use a polylactide-based capsule-shaped anisotropic channeled porous scaffold with a lumen temporarily blocked by a Teflon blocker to create a pre-vascularized cavity using the host body as a living bioreactor. After ensuring sufficient vascularization of the polymer capsule, the Teflon blocker may be removed to allow the resultant lumen to be used for subsequent islet transplantation (Fig. 1). It is further anticipated that such a prevascularized cavity may reduce the ischemia period and thereby improve the rate of successfully engrafted cells. In the current article, we aim to demonstrate the effect of an anisotropic channeled pore architecture of polylactidebased capsules on cell infiltration and vascularization. We used a similar capsule-shaped scaffold made from a medical grade surgical mesh with an isotropic regular pore structure as a control. Also, we aim to demonstrate the influence of subcutaneous space on cell infiltration and vascularization in the capsules; and we explored the greater omental pouch for comparison. The polymeric capsules were prepared using the Dip TIPS (dipping followed by thermally-induced phase separation) method recently developed in our lab (Kasoju et al. 2014, 2015). The capsules were implanted in healthy Brown Norway rats and after 4 weeks, they were excised and analyzed by histochemical and immuno-histochemical stains to assess the biocompatibility and vascular response.

\section{Methods}

\section{Materials}

High molecular weight poly(L-lactide-co- $\varepsilon$ caprolactone) (PLCL, $\mathrm{M}_{\mathrm{w}}=316000 \mathrm{~g} / \mathrm{mol}$ and $\mathrm{M}_{\mathrm{n}}=$ $120000 \mathrm{~g} / \mathrm{mol}$ ) were synthesized by ring-opening 


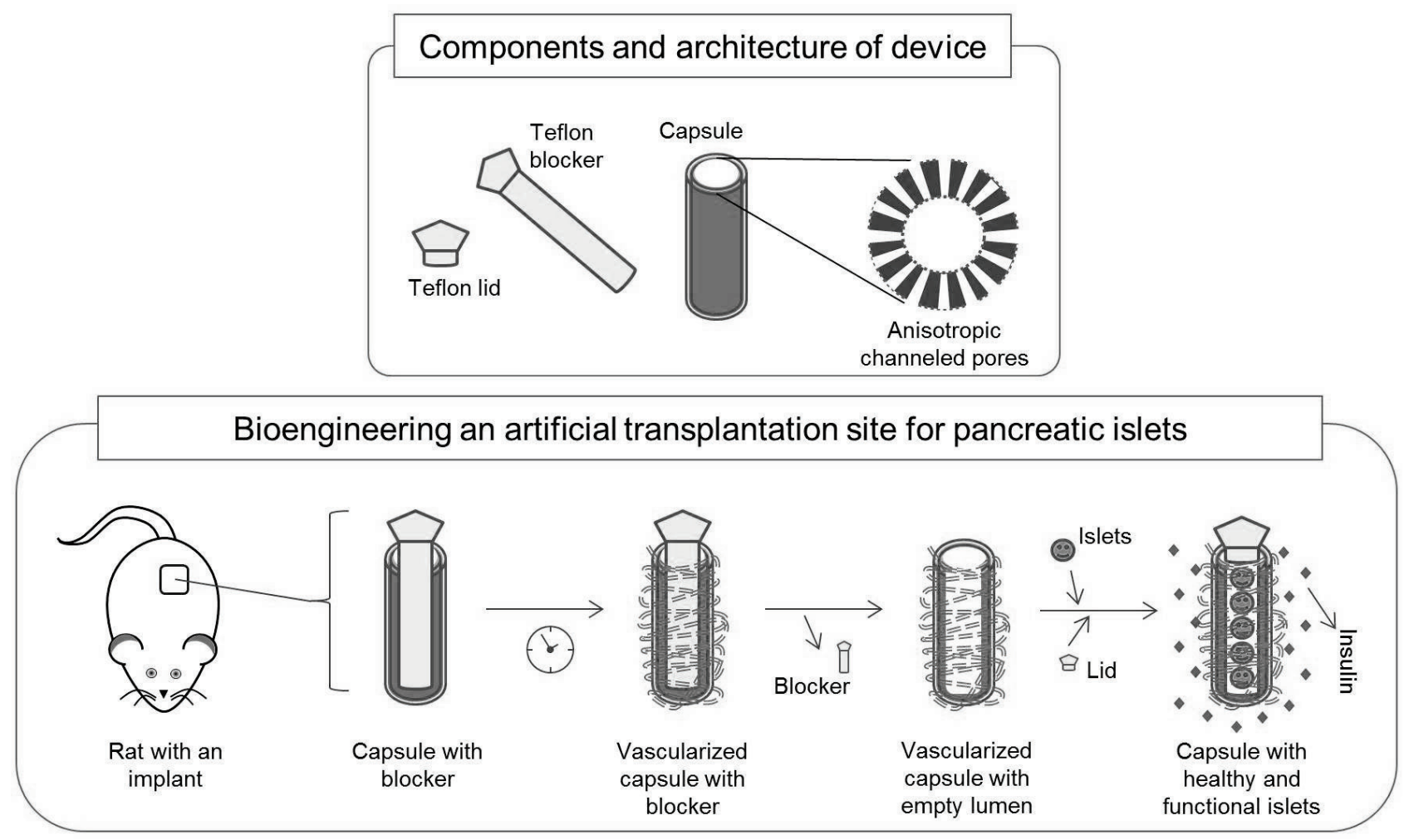

Fig. 1. Components of an implant and the hypothesis of bioengineering an artificial transplantation site for pancreatic islets. A capsule with the lumen closed by a Teflon blocker will be implanted in an animal. After a few weeks of implantation, the capsule with its anisotropic channeled oriented interconnected pores will allow the guided infiltration of host cells, including vascular endothelial cells, and thereby create a vascularized capsule. Subsequently, the Teflon block will be removed, the empty lumen will be filled with freshlyisolated healthy islets and the opening of the capsule will be closed with a Teflon lid. The transplanted islets are expected to feel comfortable with the pre-vascularized artificial microenvironment and are therefore anticipated to release insulin.

co-polymerization of the corresponding monomers (L-lactide, $\varepsilon$-caprolactone) in the presence of $\operatorname{Tin}(\mathrm{II})$ 2-ethylhexanoate as a catalyst in bulk as previously described (Kubies et al. 2000). Capsules based on a nonabsorbable Polyamide monofilament based mesh were purchased from ELLA-CS, Czech Republic, and were used as the control capsules. We purchased hematoxylin and eosin from Roche s.r.o., Czech Republic, rabbit antiCD31 and PECAM1 (cluster of differentiation 31 and platelet endothelial cell adhesion molecule-1) polyclonal antibody from Acris Antibody GmbH, Germany, biotinylated goat anti-rabbit $\operatorname{IgG}$ antibody $(\mathrm{H}+\mathrm{L})$ included in Vectastain Elite ABC Reagent, R.T.U. (Ready-to-Use) kit from Vector Laboratories, California, USA, and paraffin (histowax $56-58{ }^{\circ} \mathrm{C}$ ) from Bamed s.r.o., Czech Republic. All other reagents and chemicals, unless otherwise stated, were obtained from P-Lab a.s., LachNer s.r.o. and Sigma-Aldrich Czech Republic, and were used as received. Brown Norway rats (male, 230-270 g, aged between 2-3 months) were purchased from Charles River, Germany.

\section{Preparation of polymer capsules}

The experimental setup and the process of PLCL capsule fabrication are presented in Figure 2 (Kasoju et al. 2014, 2015). The setup includes (i) a template (a solid cylindrical thermally-conductive metallic bar with $4 \times$ $40 \mathrm{~mm}$ diameter and height respectively), (ii) a conductor (a solid cylindrical thermally-conductive metallic block with $30 \times 30 \mathrm{~mm}$ diameter and height respectively), and (iii) a reservoir (a hollow cylindrical thermally non-conductive non-metallic tube with $30 \times 120 \mathrm{~mm}$ inner diameter and height respectively). The template was attached at one end to the conductor, which was in turn bolted into the reservoir (template $\rightarrow$ conductor $\rightarrow$ reservoir).

The template was dipped into a PLCL solution ( $5 \% \mathrm{w} / \mathrm{v}$, in 1,4-dioxane), and the reservoir was filled with a dry ice/ethanol cooling mixture (which gives a cooling temperature of $-80^{\circ} \mathrm{C}$ ) to freeze the PLCL solution in the immediate vicinity of the template bar. This freezing step is hereby referred to as quenching. The quenching time was counted from the point when the freezing of the PLCL solution was first observed over the template. After $30 \mathrm{~s}$ of quenching, the template with 
phase separated PLCL-dioxane phases was removed from the PLCL solution and was allowed to coarsen for $60 \mathrm{~s}$. They were then dipped in dioxane for the second quenching, and the time was counted from the point of dipping. After $30 \mathrm{~s}$, the original PLCL-dioxane phases with an external dioxane phase were coarsened for $60 \mathrm{~s}$. The coarsened samples were then subjected to freezedrying to yield the porous capsules.

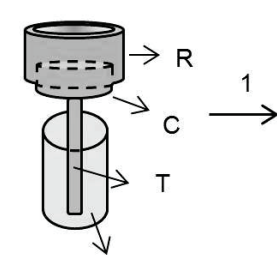

Polymer solution

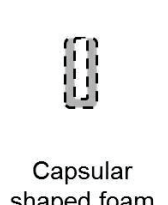

shaped foam

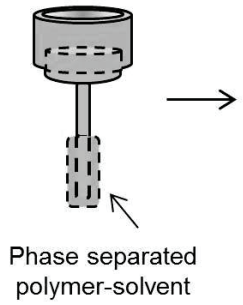

polymer-solvent

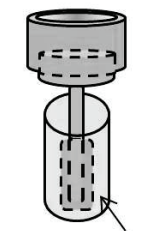

Pure solvent

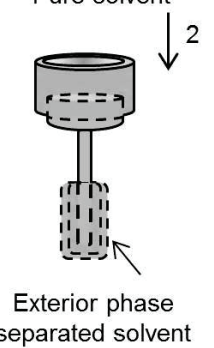

Fig. 2. The components of the setup and the process of capsule preparation. The setup includes a reservoir tube (R), a conducting block (C) and a template bar (T). The T-bar was dipped in a polymer solution and the R-tube was filled with a cooling mixture to freeze the polymer solution in the immediate vicinity of the T-bar (1). It was then immediately dipped in the solvent to form an exterior layer of frozen solvent (2). The T-bar with the frozen layers was lyophilized overnight (3). The freezedried capsular foams are finally collected from the T-bar (4).

\section{Animal care and use}

The current study was carried out in accordance with the recommendations for the care and use of laboratory animals of the Institute for Clinical and Experimental Medicine (IKEM), Prague, Czech Republic. All the protocols related to this study were approved by the Animal Care Committee of the IKEM and the Ministry of Health, Czech Republic. Experimental animals were maintained according to the European Convention on Animal Care in a controlled temperature, humidity and 12/12 light/dark regimen, with free access to food pellets and water. All the surgical procedures were done under total anesthesia induced by a mixture of medetomidine and ketamine injected intramuscularly. At the end of the study, the test samples were excised from the animals under general anesthesia induced by a mixture of medetomidine and ketamine injected intramuscularly, and subsequently, the animals were euthanized using exsanguination. Throughout the entire study, all efforts were made to minimize suffering by the animals.

\section{Surgery and capsule implantation}

Anisotropic channeled porous PLCL capsules were used as the test samples, while isotropic regular porous Polyamide capsules were used as the control samples. Prior to the implantation, all the test capsules were sterilized in a $70 \%$ ethanol for $1 \mathrm{~h}$ and were then thoroughly washed with sterile distilled water. The control capsules, sterilized by gamma radiation by the manufacturer, were used without any further treatment. Both the test and the control capsules were implanted under the skin and in the greater omentum of Brown Norway rats. After insertion of the implants, the incisions were sutured in two levels with 4-0 Vicryl absorbable sutures. For 4 weeks, all the animals that received the capsules were carefully monitored for any signs of visible inflammation or other pathological reactions both in general and in particular at the site of implantation.

\section{Histochemical and immunohistochemical analysis}

After 4 weeks of implantation, the rats were euthanized and all the capsules were harvested and analyzed by histochemical and immuno-histochemical stains. For this purpose, the implants were washed in PBS, fixed with $4 \%$ formaldehyde in PBS overnight, dehydrated through a graded series of ethanol, embedded in paraffin and sectioned at a thickness of $5 \mu \mathrm{m}$. The sections were then de-paraffinized, rehydrated with a graded series of ethanol and stained with the following stains as per the standard protocols: hematoxylin and eosin (H\&E, to display the cytoplasmic and nuclear features of the tissue), Masson's trichrome (TRI, to display extracellular matrix components, particularly collagen) and anti-CD31 antibody (CD31, to display the presence of vascular endothelial cells) (Fischer et al. 2008, Pusztaszeri et al. 2006).

\section{Results}

\section{Typical morphological features of the capsules}

The gross appearance of the PLCL capsule (the test sample) is presented in Figure 3a. The inner lumen of the capsule has a diameter of $4 \mathrm{~mm}$ and a length of $20 \mathrm{~mm}$, and thus the capsule provides a volume of $251 \mathrm{~mm}^{3}$, which is sufficient to accommodate $250 \mu \mathrm{l}$ of islet suspension. A schematic of various sections of the 
capsule analyzed by SEM is shown in Figure 3b. A cross section of the capsule revealed the channeled pores that were long and nearly continuous from one end to the other (Fig. 3c). The thickness of the capsule wall was found to be $600-650 \mu \mathrm{m}$. A longitudinal section (refer to the dotted line in Figure 3c) showed the honeycomb network of the pores with sizes as low as $\sim 10 \mu \mathrm{m}$ and as high as $\sim 80 \mu \mathrm{m}$ (Fig. 3d). The outer surface exhibited pores $80-100 \mu \mathrm{m}$ in size (Fig. 3e), and the inner surface revealed pores $5-10 \mu \mathrm{m}$ in size (Fig. $3 \mathrm{f}$ ). It was thus evident that the capsule possessed radially-oriented, interconnected, anisotropic and channeled pores. The gross appearance of the Polyamide-based capsule (a control sample) is presented in Figure 3g. The dimensions and volume of the inner lumen of the control capsule were similar to that of the test capsule, i.e. $4 \mathrm{~mm}$ diameter $\times 20 \mathrm{~mm}$ height and a volume of $251 \mathrm{~mm}^{3}$. A stereo microscope (SZ61-TR, Olympus) image of the control capsule showing the isotropic regular pores with a size of $860 \pm 45 \mu \mathrm{m}$ is presented in Figure $3 \mathrm{~h}$.

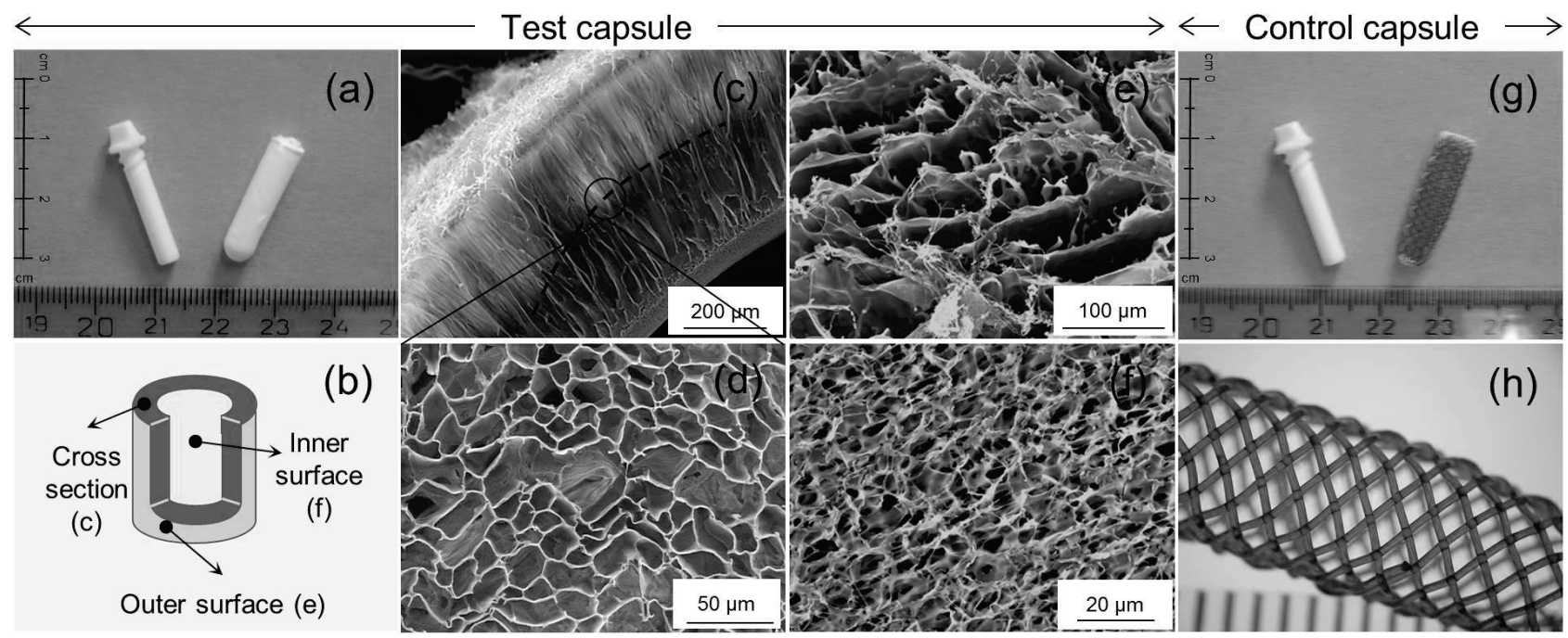

Fig. 3. Typical morphological features of the test and control capsules. Macroscopic (a) and scanning electron microscopy images of the test capsule (c-f), including a scheme of various sections of the capsule (b). Macroscopic ( $\mathbf{g})$ and light microscopy (h) images of the control capsule. A Teflon bar can also be seen in the macroscopic images (a, g). The test capsule has anisotropic channeled interconnected pores in contrast to the control capsule, which has isotropic regular pores.

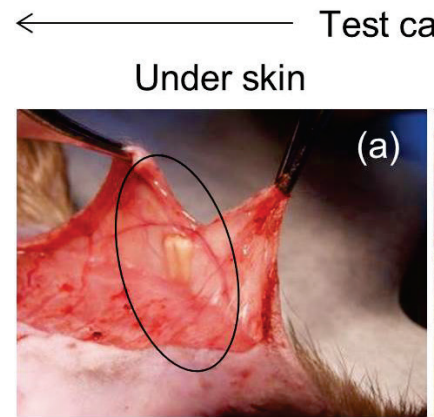

$\left(a^{\prime}\right)$
)

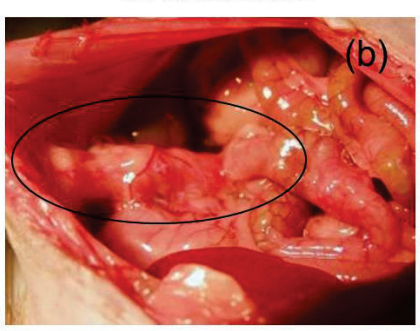

(b')

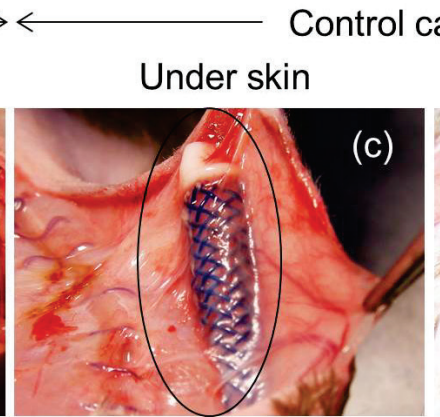

(c')

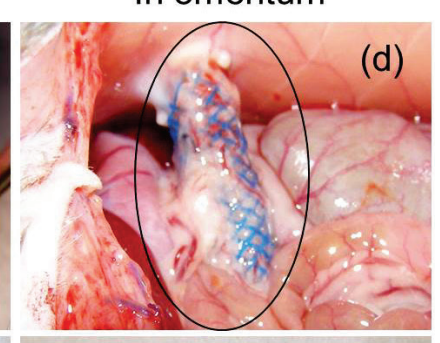

(d')
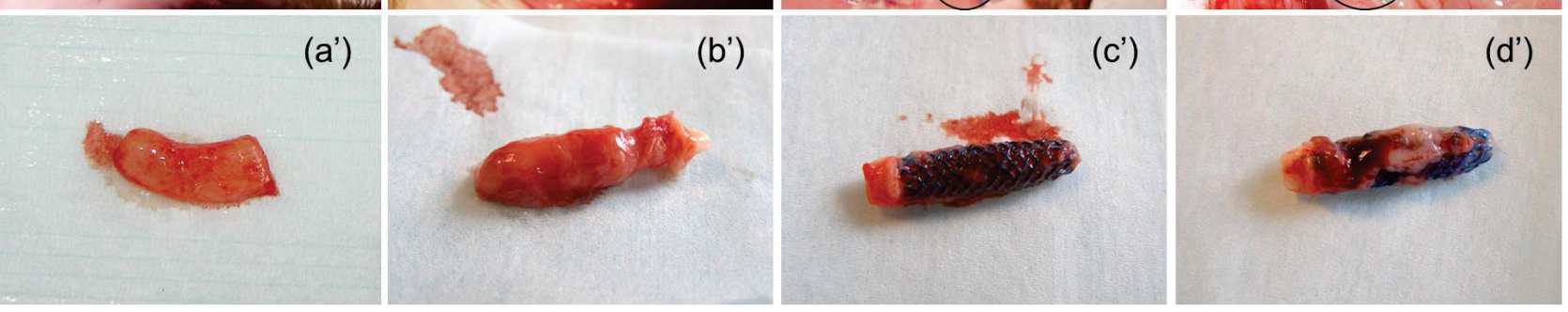

Fig. 4. Digital camera (SP500 UZ, Olympus; auto-mode) images of a test capsule under the skin (a, $\left.\mathbf{a}^{\prime}\right)$, in the greater omentum $\left(\mathbf{b}, \mathbf{b}^{\prime}\right)$, and a control capsule under the skin $\left(\mathbf{c}, \mathbf{c}^{\prime}\right)$ and in the greater omentum $\left(\mathbf{d}, \mathbf{d}^{\prime}\right)$. The location of the implants is marked by a circle. It was evident that both the test and the control capsules were anastomosed with the host body without any detectable signs of inflammation or fibrotic response. The white tissue seen on the capsules in the omentum was omental fat tissue. The blue color of the control capsules was inherent. 
Post-operative behavior, excision of capsules and macroscopic observations

All of the rats survived until the end of the experiment and showed normal behavior. There was no post-operative infection or any evidence of a foreign body reaction. The rats showed excellent clinical recovery during the post-operative period, both in general and in particular at the site of implantation. After 4 weeks, the animals were cut open to check the integrity and health of the capsules (Figs 4a-4d). The capsules, at all locations, were effectively anastomosed with the host body, without showing any visible signs of inflammation or other pathological complications. The capsules were carefully dissected out of the animal bodies (Figs $4 a^{\prime}-4 d$ '). Macroscopic observations revealed that the host tissue had adequately penetrated and covered the capsules. The integrity of the newly-formed tissue through or surrounding the capsules was confirmed by the typical sound of a vacuum generated when the Teflon blockers were removed. Notably, the capsules implanted in the omentum, both test and control, were rich in vessels in comparison to the capsules implanted subcutaneously. In addition, the capsules implanted into the greater omentum were associated with omental fat.

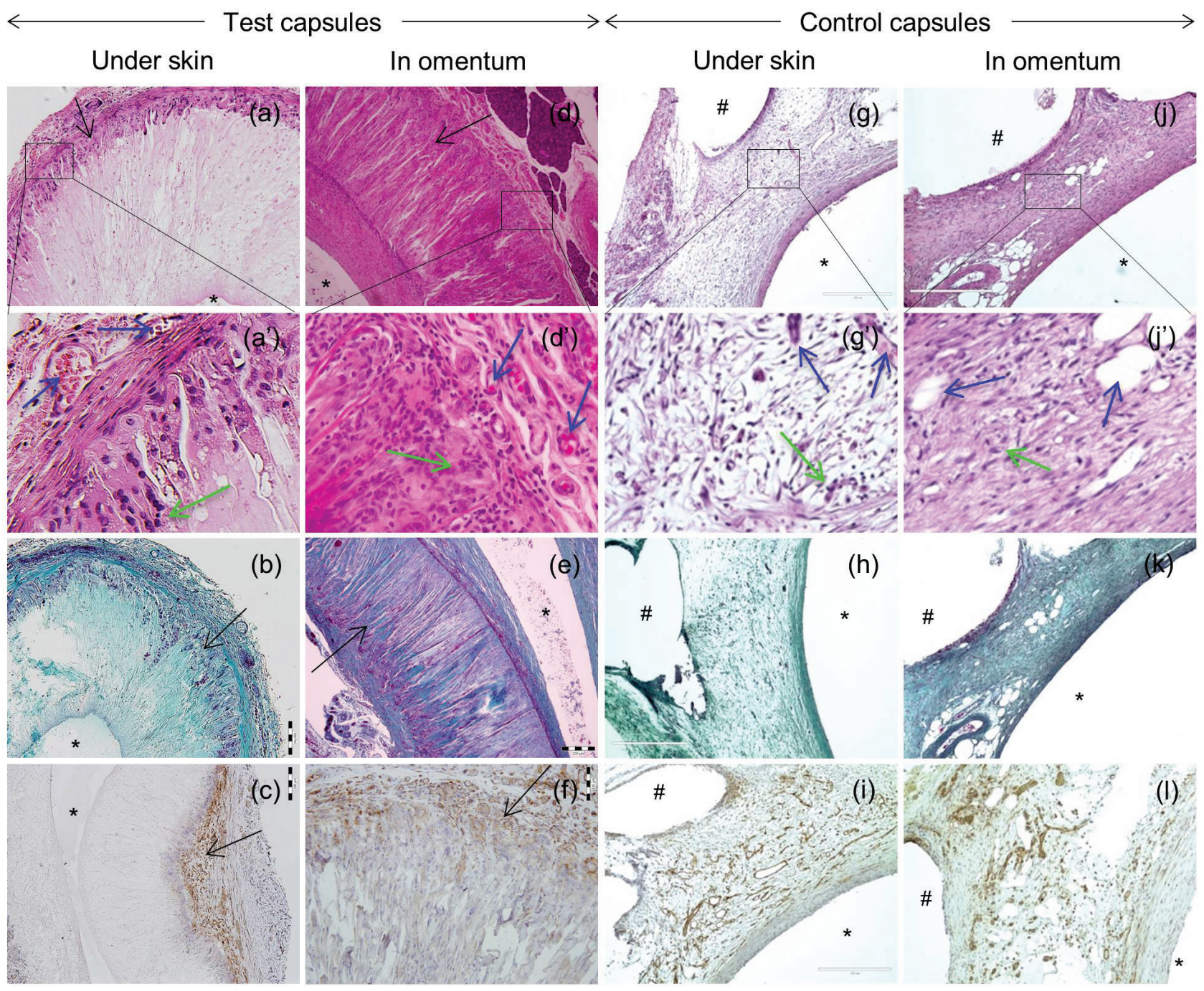

Fig. 5. Hematoxylin and eosin ( $\mathrm{HE}, \mathbf{a}, \mathbf{d}, \mathbf{g}, \mathbf{j}$, and $\mathbf{a}^{\prime}, \mathbf{d}^{\prime}, \mathbf{g}^{\prime}, \mathbf{j}^{\prime}-200 \times$ magnification), Masson's trichrome (TRI, b, e, h, k) and antiCD31 antibody (CD31, $\mathbf{c}, \mathbf{f}, \mathbf{i}, \mathbf{I})$ stained cross sections of the test and control capsules, both under the skin and in the greater omentum. As evident from HE and TRI staining, the host cell infiltration was guided in the anisotropic channeled porous test capsules in contrast to the isotropic random porous control capsules, and cell infiltration was better supported in the omentum than under the skin. As revealed by CD31 staining, neovascularization was suboptimal in all cases and vasculature was present only on the surface of the capsules. Black arrows indicate the direction of host cell infiltration, while blue arrows indicate the blood vessels, green arrows indicate the giant cells, * denotes the inner lumen created by the Teflon blocker and \# indicates the empty space left behind by the fibers of the control capsule. All the images were taken at $100 \times$ magnification unless otherwise noted. 
Histochemical and immunohistochemical staining and microscopic observations

In the HE stained images (Figs 5a, 5d, 5g, 5j), the nuclei were stained deep blue to purple (by hematoxylin), whereas the cytoplasm and extracellular matrix had varying degrees of pink staining (by eosin). The presence of cells was clearly evident in all the capsules. A careful analysis of the images revealed four compelling observations. First, in no cases there were any significant numbers of cells in or around the capsules with an unusual size or shape that resembled inflammatory cells. Second, there was a very thin layer of tissue surrounding the capsules, especially the test, both in the omentum and under the skin. Third, both in the omentum and under the skin, the test capsules promoted guided cell infiltration through their channeled pores, in comparison to the control capsules that supported noncontrolled cell infiltration and a randomly organized network of fibrous tissue. And fourth, with both the test and the control, the capsules implanted in the omentum supported higher cell infiltration than the capsules implanted under the skin.

The images of Masson's trichrome stained sections revealed blue or green collagen, light red or pink cytoplasm and dark brown or black nuclei (Figs 5b, 5e, $5 \mathrm{~h}, 5 \mathrm{k})$. The results were in good agreement with the HE staining showing guided cell infiltration in test capsules and random cell infiltration in control capsules, and a high density of cells in the omental capsules and a poor or low density of cells in the subcutaneous capsules. On the other hand, the paraffin sections stained with antiCD31 antibody showed the brown colored vascular network or endothelial cell boundaries (Figs 5c, 5f, 5i, 51). Similar sections used as negative controls for antiCD31 staining did not show any brown colored cells (data not shown). As shown in Figures 5c, 5f, 5i, 51, there were numerous vessels located mostly on the external surface of the capsules at all locations. And, in contrast to the macroscopic view where the vessel density seemed low in the subcutaneous capsules (Fig. 4), multiple vessels were also detected in the capsules implanted subcutaneously (Figs 5c and 5i).

\section{Discussion}

An optimal extra-hepatic site for islet transplantation should a) provide physical confinement, b) protect against mechanical stress, c) be accessible with minimum invasion, d) induce low or no activation of the immune system, and last but not least, e) promote rapid revascularization to reduce the ischemia period to as short as possible (Smink et al. 2013). The human body does not contain an environment that meets the aforesaid ideal requirements for islet transplantation. Therefore, biomaterial-based bioengineering of an artificial transplantation site for pancreatic islets was proposed as early as 1996. However, the polymers used in the early attempts suffered from serious biocompatibility issues. For example, a scaffold made from polytetrafluoroethylene and implanted in the intraperitoneal cavity was associated with a strong foreign body response creating an avascular fibrous barrier (De Vos et al. 1997). Ideally, the scaffolding material for a bioengineered islet transplantation site should already be approved for human application. Thus, in the current study, we fabricated the capsules from a copolymer made of polylactide (PLA) and polycaprolactone (PCL), which are proven biocompatible materials and are U.S. Food and Drug Administration and European Conformity approved polymers (Maquet et al. 2000). Both PLA and PCL are man-made polymers, and are synthesized without any batch to batch variations, unlike natural polymers such as silk fibroin (Kasoju and Bora 2012) and polysaccharides (Bačáková et al. 2014) which suffer from serious reproducibility and variable performance issues (Langlois et al. 2009). As evident from Figure 4 and Figure 5, the PLCL capsules implanted both under the skin and in the omentum have adequately anastomosed without any serious signs of infiltration of inflammatory cells or fibrotic barrier formation. Our observations comply with the literature and reiterate the biocompatibility of the PLA and PCL polymers.

In addition to biocompatibility, the success of a scaffold in cell transplantation and tissue engineering applications highly depends on its pore properties. In the current study, as shown in Figure 3, the PLCL capsule was characterized by an anisotropic channeled porous architecture, in contrast to the isotropic regular porous structure of the control capsule. The advantages of the PLCL capsule's pore architecture over that of the control capsule was clearly evident from the histological analysis; the anisotropic channeled pores promoted the guided infiltration of cells, whereas the isotropic regular pores supported random cell infiltration (Figs 5a, 5d vs $5 \mathrm{~g}, 5 \mathrm{j}$ ). Our results are in good agreement with the literature indicating that anisotropic channeled porous scaffolds inherently promote guided cell infiltration and yield a fully-functional viable tissue without any necrotic 
core (Silva et al. 2006). However, we found that cell density, as reflected by the intensity of the HE stain, in the PLCL capsules implanted in the omentum was higher than in those capsules under the skin (Figs 5a vs 5d). But such phenomenon was also observed in the control capsules (Figs $5 \mathrm{~g}$ vs 5j). Therefore, the cell density differences in the capsules were attributed to the site of implantation rather than to the pore architecture. A poor vascular network, surgery-induced local reparative inflammation and possible mechanical stresses were the contributing factors for the poor performance of the capsules under the skin; on the other hand, the abundant vascular network and protective environment contributed to the success of the implant in the omental pouch. Similar observations were reported when the ureteral decellularized matrix (Matsunuma et al. 2006) PLA scaffolds seeded with hepatocytes (Lee et al. 2003) were implanted in the omentum and under the skin of model rats.

Finally, the necessity of having adequate vascularization for the success of any scaffold or device for islet transplantation applications can be derived from the fact that the islets consume $5-15 \%$ of the total pancreatic blood volume in order to meet their high metabolic rate, although they constitute only $1-2 \%$ of the total pancreatic cell mass (Olerud et al. 2009). In the current study, the presence of a vascular network in and around the PLCL capsules that were implanted both under the skin and in the omentum were analyzed after 4 weeks. From the gross observations, as evident from Figure 4, the capsules implanted in the omentum had a dense vasculature in comparison to the capsules under the skin. These observations were supported by the dynamic contrast-enhanced magnetic resonance imaging analysis [data not shown, imaging was performed as per (Kriz et al. 2011)]. As outlined in the previous paragraph, the differences in the vascular response around the PLCL capsules may be attributed to the varying level of the natural vasculature in the omentum and under the skin. For a better understanding, we analyzed the capsules using anti-CD31 immuno-histochemical staining. As shown in Figure 5, the presence of numerous vessel-like structures was evident at all locations; however, the vasculature was located mostly on the external surface of the capsules and did not succeed in penetrating through the capsule until the edge of the lumen. Such observations were not surprising given the fact that the PLCL capsule was bio-inert and had no additional proangiogenic factors. This was in accordance with literature wherein it was demonstrated that pristine scaffolds made of polymers such as poly(L-lactide-co-glycolide), poly(caprolactone), etc., failed to achieve adequate vascularization without the inclusion of pro-angiogenic factors (Lindhorst et al. 2010).

\section{Conclusions}

For the first time, in the current study, we describe the use of polylactide-based anisotropic channeled porous capsules for bioengineering a vascularized microenvironment, using the host body as a living bioreactor, for potential applications in islet transplantation. The anisotropic channeled porous test capsule and isotropic regular porous control capsule were implanted under the skin and in the greater omentum in Brown Norway rats. For 4 weeks, the post-operative recovery and behavior of the rats was satisfactory and normal. The hematoxylin and eosin and Masson's trichrome staining images showed that a) there was no significant infiltration of inflammatory cells, b) there was minimal or no pathological fibrotic response around the capsules, c) the infiltration of the host's connective tissue cells was guided through the channeled pores of the test capsule in contrast to random cell infiltration in the regular porous control capsule, and d) the capsules implanted in the greater omentum appeared to have relatively superior cell infiltration over the capsules implanted under the skin. Furthermore, anti-CD31 immuno-histochemistry revealed that the vascular network was present at the implant site, but mostly on the external surface of the capsules. Taken together, the current study is a significant step forward in the field of biomaterial-based extra-hepatic islet transplantation.

\section{Conflict of Interest}

There is no conflict of interest.

\section{Acknowledgements}

The study was funded by the Ministry of Education, Youth and Sports, Czech Republic (grant number EE2.3.30.0029 - as project BIOPOL, awarded to IMC, Prague), by the Ministry of Health, Czech Republic (grant number 00023001 - as Institutional Support for the Development of Research Organization, and grant number NT14240-3, awarded to ICEM, Prague), and by the European Regional Development Fund (grant number CZ.1.05/1.1.00/02.0109 - as project BIOCEV, awarded to IMC, Prague). 


\section{References}

BAČÁKOVÁ L, NOVOTNÁ K, PAŘÍZEK M: Polysaccharides as cell carriers for tissue engineering: The use of cellulose in vascular wall reconstruction. Physiol Res 63 (Suppl 1): S29-S47, 2014.

BRATLIE KM, YORK RL, INVERNALE MA, LANGER R, ANDERSON DG: Materials for diabetes therapeutics. Adv Healthcare Mater 1: 267-284, 2012.

CAIAZZO R, GMYR V, HUBERT T, DELALLEAU N, LAMBERTS R, MOERMAN E, KERR-CONTE J, PATTOU F: Evaluation of alternative sites for islet transplantation in the minipig: Interest and limits of the gastric submucosa. Transplant Proc 39: 2620-2623, 2007.

CANTARELLI E, MELZI R, MERCALLI A, SORDI V, FERRARI G, LEDERER CW, MRAK E, RUBINACCI A, PONZONI M, SITIA G, GUIDOTTI LG, BONIFACIO E, PIEMONTI L: Bone marrow as an alternative site for islet transplantation. Blood 114: 4566-4574, 2009.

CARLSSON PO, PALM F, ANDERSSON A, LISS P: Chronically decreased oxygen tension in rat pancreatic islets transplanted under the kidney capsule. Transplantation 69: 761-766, 2000.

De VOS P, HILLEBRANDS J-L, DE HAAN BJ, STRUBBE JH, VAN SCHILFGAARDE R: Efficacy of a prevascularized expanded polytetrafluoroethylene solid support system as a transplantation site for pancreatic islets1. Transplantation 63: 824-830, 1997.

FISCHER AH, JACOBSON KA, ROSE J, ZELLER R: Hematoxylin and eosin staining of tissue and cell sections. CSH Protoc 2008: pdb.prot4986, 2008.

KASOJU N, BORA U: Silk fibroin in tissue engineering. Adv Healthcare Mater 1: 393-412, 2012.

KASOJU N, KUBIES D, KUMOREK MM, KRIZ J, FABRYOVA E, MACHOVA L, KOVAROVA J, RYPACEK F: Dip tips as a facile and versatile method for fabrication of polymer foams with controlled shape, size and pore architecture for bioengineering applications. PLoS One 9: e108792, 2014.

KASOJU N, KUBIES D, SEDLAČÍK T, JANOUŠKOVÁ O, KOUBKOVÁ J, KUMOREK MM, RYPACEK F: Polymer scaffolds with fully open porous surfaces for effective cell infiltration in tissue engineering applications fabricated by thermally induced phase separation. Submitted, 2015.

KORSGREN O, LUNDGREN T, FELLDIN M, FOSS A, ISAKSSON B, PERMERT J, PERSSON NH, RAFAEL E, RYDEN M, SALMELA K, TIBELL A, TUFVESON G, NILSSON B: Optimising islet engraftment is critical for successful clinical islet transplantation. Diabetologia 51: 227-232, 2008.

KRIZ J, JIRAK D, KOBLAS T, VILK G, GIRMAN P, HAJEK M, SAUDEK F: Dynamic contrast-enhanced magnetic resonance imaging as a tool to monitor the blood supply to an artificial cavity used as a site for islet transplantation in rats. Transplant Proc 43: 3226-3230, 2011.

KROL S, Del GUERRA S, GRUPILlO M, DIASPRO A, GLIOZZI A, MARCHETTI P: Multilayer nanoencapsulation. New approach for immune protection of human pancreatic islets. Nano Lett 6: 1933-1939, 2006.

KUBIES D, RYPACEK F, KOVAROVA J, LEDNICKY F: Microdomain structure in polylactide-block-poly(ethylene oxide) copolymer films. Biomaterials 21: 529-536, 2000.

LANGLOIS G, DUSSEAULT J, BILODEAU S, TAM SK, MAGASSOUBA D, HALLE JP: Direct effect of alginate purification on the survival of islets immobilized in alginate-based microcapsules. Acta Biomater 5: 34333440, 2009.

LEE H, CUSICK RA, UTSUNOMIYA H, MA PX, LANGER R, VACANTI JP: Effect of implantation site on hepatocytes heterotopically transplanted on biodegradable polymer scaffolds. Tissue Eng 9: 1227-1232, 2003.

LEITÃO C, CURE P, THARAVANIJ T, BAIDAL D, ALEJANDRO R: Current challenges in islet transplantation. Curr Diab Rep 8: 324-331, 2008.

LINDHORST D, TAVASSOL F, VON SEE C, SCHUMANN P, LASCHKE MW, HARDER Y, BORMANN K-H, ESSIG H, KOKEMÜLLER H, KAMPMANN A, VOSS A, MÜLHAUPT R, MENGER MD, GELLRICH NC, RÜCKER M: Effects of vegf loading on scaffold-confined vascularization. $J$ Biomed Mater Res A 95A: 783-792, 2010. 
MA M, CHIU A, SAHAY G, DOLOFF JC, DHOLAKIA N, THAKRAR R, COHEN J, VEGAS A, CHEN D, BRATLIE KM, DANG T, YORK RL, HOLLISTER-LOCK J, WEIR GC, ANDERSON DG: Core-shell hydrogel microcapsules for improved islets encapsulation. Adv Healthcare Mater 2: 667-672, 2013.

MAQUET V, MARTIN D, MALGRANGE B, FRANZEN R, SCHOENEN J, MOONEN G, JÉRÔME R: Peripheral nerve regeneration using bioresorbable macroporous polylactide scaffolds. J Biomed Mater Res 52: 639-651, 2000.

MATSUNUMA H, KAGAMI H, NARITA Y, HATA K, ONO Y, OHSHIMA S, UEDA M: Constructing a tissueengineered ureter using a decellularized matrix with cultured uroepithelial cells and bone marrow-derived mononuclear cells. Tissue Eng 12: 509-518, 2006.

OLERUD J, JOHANSSON Å, CARLSSON P-O: Vascular niche of pancreatic islets. Expert Rev Endocrinol Metab 4: 481-491, 2009.

PEREZ VL, CAICEDO A, BERMAN DM, ARRIETA E, ABDULREDA MH, RODRIGUEZ-DIAZ R, PILEGGI A, HERNANDEZ E, DUBOVY SR, PAREL JM, RICORDI C, KENYON NM, KENYON NS, BERGGREN PO: The anterior chamber of the eye as a clinical transplantation site for the treatment of diabetes: A study in a baboon model of diabetes. Diabetologia 54: 1121-1126, 2011.

PUSZTASZERI MP, SEELENTAG W, BOSMAN FT: Immunohistochemical expression of endothelial markers cd31, cd34, von willebrand factor, and fli-1 in normal human tissues. J Histochem Cytochem 54: 385-395, 2006.

SHAPIRO AMJ, LAKEY JRT, RYAN EA, KORBUTT GS, TOTH E, WARNOCK GL, KNETEMAN NM, RAJOTTE $\mathrm{RV}$ : Islet transplantation in seven patients with type 1 diabetes mellitus using a glucocorticoid-free immunosuppressive regimen. $N$ Engl J Med 343: 230-238, 2000.

SILVA MMCG, CYSTER LA, BARRY JJA, YANG XB, OREFFO ROC, GRANT DM, SCOTCHFORD CA, HOWDLE SM, SHAKESHEFF KM, ROSE FRAJ: The effect of anisotropic architecture on cell and tissue infiltration into tissue engineering scaffolds. Biomaterials 27: 5909-5917, 2006.

SMINK AM, FAAS MM, DE VOS P: Toward engineering a novel transplantation site for human pancreatic islets. Diabetes 62: 1357-1364, 2013.

STAGNER JI, RILO HL, WHITE KK: The pancreas as an islet transplantation site. Confirmation in a syngeneic rodent and canine autotransplant model. JOP 8: 628-636, 2007.

SVENSSON J, LAU J, SANDBERG M, CARLSSON PO: High vascular density and oxygenation of pancreatic islets transplanted in clusters into striated muscle. Cell Transplant 20: 783-788, 2011.

TATARKIEWICZ K, HOLLISTER-LOCK J, QUICKEL RR, COLTON CK, BONNER-WEIR S, WEIR GC: Reversal of hyperglycemia in mice after subcutaneous transplantation of macroencapsulated islets. Transplantation 67 : 665-671, 1999.

VARDANYAN M, PARKIN E, GRUESSNER C, RODRIGUEZ RILO HL: Pancreas vs. Islet transplantation: A call on the future. Curr Opin Organ Transplant 15: 124-130, 2010.

WOHLRAB F, WILKE B, SCHMIDT S, COSSEL L: Syngeneic transplantation of rat pancreatic islets into the spleen. Light and electron microscopical findings. Int J Pancreatol 3: 59-72, 1988. 